\title{
GRAVITATIONAL COLLAPSE OF HOMOGENEOUS SCALAR FIELDS
}

\author{
ROBERTO GIAMBÒ
}

\begin{abstract}
Conditions under which gravity coupled to self interacting scalar field determines singularity formation are found and discussed. It is shown that, under a suitable matching with an external space, the boundary, if collapses completely, may give rise to a naked singularity. Issues related to the strength of the singularity are discussed.
\end{abstract}

\section{INTRODUCTION}

The gravitational collapse of scalar fields in classical General Relativity has been widely studied in literature. Its role in understanding the machinery ruling singularities' causal structure - at least in spherical symmetry - was clear since 90's, when models of scalar field collapse exhibiting a naked singularity, and therefore violating the so-called Penrose's Cosmic Censorship Conjecture [18], were found numerically by Choptuik [1] and analytically by Christodoulou [2].

In these pioneering works the scalar field is massless and free, that means that the Lagrangian function of the field does not contain any mass or potential terms. Recently, Hertog, Horowitz and Maeda [10] found a class of potentials where smooth initial data evolve to give rise to a naked singularity, although energy conditions may be violated. Moreover, in a recent paper, Goswami and Joshi [8] studied collapse of self interacting scalar field, under a homogeneity assumption, considering a class of models where divergence of energy density near the singularity is assumed to satisfy a power-law relation.

As is well known scalar field have not been observed although their fundamental relevance in cosmology. Observational cosmology is indeed probably the unique field in which we can hope to obtain information on the scalar field potential, and several works go in this direction, as e.g. [15] (and references therein) in which the analysis of transition from matter domination to dark energy domination is taken into account, although the expanding case is obviously considered there.

In the present paper, we consider homogeneous scalar fields collapsing models in general, characterizing the class of potentials determining singularity formation. A crucial role in determining the causal structure is played by the existence, or not, of apparent horizon during the evolution. Since the singularity is (of course) synchronous, the behavior is quite different from what happens in many examples of matter models exhibiting a central naked singularity [5, 7]. Here, instead, it may be that the singularity located at the boundary of the "ball" of scalar field can be naked. Therefore, the scalar field solution must be matched with a suitable external solution and behavior of radial geodesic in the external solution must be studied accordingly.

Date: April 2005 (revised version). 
Scalar field solution under homogeneity assumption is introduced in Section 2 In Section 3 necessary and sufficient conditions for singularity formation are stated, together with conditions which forbid apparent horizon formation during the evolution. In the latter case, a matching with anisotropic generalization of de Sitter spacetime [6] is performed (Section 4), and naked singularity existence is proved - and its gravitational strength discussed - in Section 5 Reasons for our choice of exterior region are discussed in final Section 6 together with overall conclusions.

\section{THE SOLUTION}

The general scalar field spacetime is a spacetime $\left(\mathcal{M}, g_{\mu \nu}\right)$ satisfying Einstein field equations for the energy-momentum tensor

$$
T_{\mu \nu}=\partial_{\mu} \phi \partial_{\nu} \phi-\left(\frac{1}{2} g^{\alpha \beta} \partial_{\alpha} \phi \partial_{\beta} \phi+V(\phi)\right) g_{\mu \nu},
$$

where $\phi$ is a scalar function on $\mathcal{M}$ and $V$ is the potential. We consider the case of a spherically symmetric spacetime where the gradient of $\phi$ is timelike, which allows us to choose a comoving gauge such that $\phi$ is a function of comoving time only, and the metric and the energy-momentum tensor can be respectively written in the form [12]

$$
\mathrm{d} s^{2}=-\mathrm{d} t^{2}+a^{2}(t) \mathrm{d} r^{2}+a^{2}(t) r^{2} \mathrm{~d} \Omega^{2},
$$

and

$$
T_{\nu}^{\mu}=\operatorname{diag}(-\epsilon, p, p, p)
$$

Equations (2.1)-2.2) imply that, in the above expression, the energy density $\epsilon$ and the stress $p$ can be written in terms of the field and the potential as

$$
\epsilon=\frac{1}{2} \dot{\phi}^{2}+V(\phi), \quad p=\frac{1}{2} \dot{\phi}^{2}-V(\phi)
$$

where the dot denotes differentiation with respect to $t$.

Field equations reduce to

$$
\begin{aligned}
& \dot{a}^{2}=\frac{8 \pi}{3} a^{2} \epsilon, \\
& \frac{\mathrm{d}}{\mathrm{d} t}\left(a^{3} \dot{\phi}\right)=-a^{3} V^{\prime}(\phi) .
\end{aligned}
$$

in the unknown functions $a(t) \geq 0$ and $\phi(t)$. The potential $V(\phi)$ has to be considered as a constitutive function that, analogously to what happen in the elastic-solid matter case [13], establishes a relation between energy and stresses. In particular, (2.6) is Klein-Gordon equation $g^{\mu \nu} \nabla_{\mu} \partial_{\nu} \phi=V^{\prime}(\phi)$, obviously equivalent to the Bianchi identity .

We will take initial data at an initial time, say $t=0$ :

$$
a(0)=a_{0}, \quad \phi(0)=\phi_{0}, \quad \dot{\phi}(0)=\dot{\phi}_{0} .
$$

The field evolves collapsing until it possibly reaches the singular state at $a=0$. We will consequently consider cases where $a$ is a monotonically decreasing function of $t$. 
This fact implies that, for instance, the energy density $\epsilon$ can be seen as a function of $a$, and can be written as

$$
\epsilon=\frac{3}{8 \pi}\left(\frac{\psi(a)}{a}\right)^{2},
$$

where $\psi \in C^{\infty}\left(\left(0, a_{0}\right),(0,+\infty)\right)$. Note that, in principle, we don't make assumption on the behavior of $\psi(a)$ as $a \rightarrow 0^{+}$but, in order to study a physically meaningful singularity, we will assume that

$$
\lim _{a \rightarrow 0^{+}} \frac{\psi(a)}{a}=+\infty
$$

Using (2.8), equation (2.5) takes the form

$$
\dot{a}=-\psi(a),
$$

where the sign choice is determined by the positive sign of $\psi(a)$ and the fact that we are considering a collapsing situation. Therefore, using initial condition (2.7), the following proposition easily follows.

Proposition 2.1. The metric (2.2) is determined by the function

$$
a(t)=F^{-1}\left(F\left(a_{0}\right)-t\right)
$$

where $F=F(a)$ is a primitive function of $1 / \psi(a)$.

Moreover, such a choice of $\psi$ also determines both the scalar function $\phi$ and the potential $V$ as functions of $a$. Indeed, with the positions

$$
R:=a(t) r, \quad m:=\frac{R}{2}\left(1-g^{\alpha \beta} \partial_{\alpha} R \partial_{\beta} R\right)=\frac{1}{2} r^{3} a(t) \dot{a}^{2}(t),
$$

using (2.4), 2.10), it is easy to see that Klein-Gordon equation (2.6) is completely equivalent to

$$
\dot{m}=-4 \pi p R^{2} \dot{R}
$$

and, in view of the above equation and (2.10) again, we can write the stress $p$ as

$$
p=-\frac{\psi^{2}(a)}{8 \pi a^{2}}\left(1+2 a \frac{\psi^{\prime}(a)}{\psi(a)}\right) .
$$

Using (2.4), 2.10), one finally finds

$$
\begin{gathered}
\left(\frac{\mathrm{d} \phi}{\mathrm{d} a}\right)^{2}=\frac{1}{4 \pi a^{2}}\left(1-a \frac{\psi^{\prime}(a)}{\psi(a)}\right), \\
V=\frac{\psi^{2}(a)}{4 \pi a^{2}}\left(1+\frac{1}{2} a \frac{\psi^{\prime}(a)}{\psi(a)}\right) .
\end{gathered}
$$

Therefore, chosen an energy profile through the function $\psi(a)$, the solution is completely expressed in function of $a$ and, consequently, of $t$. In case the function $\phi(a)$ is invertible, one can say more. Indeed, the following proposition holds. 
Proposition 2.2. If $\frac{\psi^{\prime}(a)}{\psi(a)} a<1$ in $\left(0, a_{0}\right)$, the spacetime given by (2.11) solves Einstein field equations, with the potential $V(\phi)=V(a(\phi))$, where $V(a)$ is given by (2.16), and $a(\phi)$ is the inverse function of

$$
\phi(a)=\phi_{0}+\int_{a}^{a_{0}} \sqrt{\frac{1}{4 \pi \theta^{2}}\left(1-\theta \frac{\psi^{\prime}(\theta)}{\psi(\theta)}\right)} d \theta .
$$

Remark 2.3. Since

$$
\epsilon+p=\dot{\phi}^{2}=\dot{a}^{2}\left(\frac{\mathrm{d} \phi}{\mathrm{d} a}\right)^{2},
$$

the condition $\frac{\psi^{\prime}(a)}{\psi(a)} a<1$ in $\left(0, a_{0}\right)$ can be reinterpreted by saying that the weak energy condition inequality must be strictly satisfied, that is $\epsilon+p>0$. Of course, one can invert the function $\phi(a)$ (2.17) under more general conditions also, for instance if $\epsilon+p$ vanishes for some isolate values of $a$, but in this case problems of regularity may arise.

\section{SingulARITY FORMATION}

Properties of $\psi(a)$ will be used to study singularity formation and behavior for the solutions above. The following theorem holds.

Theorem 3.1. The spacetime becomes singular in a finite amount of comoving time if and only if the function $1 / \psi(a)$ is integrable on $\left(0, a_{0}\right)$. The time of collapse is given by

$$
t_{s}=\int_{0}^{a_{0}} \frac{1}{\psi(a)} d a .
$$

Proof. If $1 / \psi(a)$ is integrable in $\left(0, a_{0}\right)$, the primitive $F(a)$ can be continuously extended to $a=0$. In this case $t_{s}=F\left(a_{0}\right)-F(0)$, and using (2.11) it follows that $a\left(t_{s}\right)=0$. Viceversa, if $a(t)=0$ for some $t_{s} \in(0,+\infty)$, then by continuity $\lim _{t \rightarrow t_{s}} a(t)=0$. Equation (2.11) again implies that $\lim _{a \rightarrow 0^{+}} F(a)=F\left(a_{0}\right)-t_{s}$, and the primitive can be extended up to $a=0$.

We will suppose hereafter that we are in the hypothesis of Theorem 3.1, so that singularity forms in a finite amount of comoving time. In order to study singularity behavior, we fix $t_{s}$ as in (3.1) and consider the apparent horizon curve $t_{h}(r)$ implicitly defined by the equation $R\left(r, t_{h}(r)\right)=2 m\left(r, t_{h}(r)\right)$. It is the boundary of the trapped region

$$
\mathcal{T}=\{(r, t): R(r, t) \leq 2 m(r, t)\} .
$$

The following result holds.

Proposition 3.2. If $\psi(a)$ is bounded in $\left(0, a_{0}\right)$, there exists $r_{b}>0$ such that, for any shell of matter $r \leq r_{b}$, no apparent horizon forms during the evolution.

Proof. Using (2.12), the inequality $R>2 m$ means that

$$
\psi(a) r<1 \text {. }
$$

Then, it suffices to choose $r_{b}<\left(\sup _{\left(0, a_{0}\right)} \psi(a)\right)^{-1}$, and, if $r \leq r_{b}$ and $t<t_{s}$, it will be $(t, r) \notin \mathcal{T}$. 
Remark 3.3. The quantity $r_{b}$ will play the role of the boundary of scalar field collapsing sphere. Note that the absence of horizon does not mean, in principle, that the singularity is naked. Indeed, if a future pointing null geodesic arising from the singularity exists, it must satisfy the ODE

$$
\frac{\mathrm{d} t}{\mathrm{~d} r}=a(t)
$$

and then it must be an increasing function of $r$. This cannot happen for $r \in\left[0, r_{b}\right)$, because the singularity is synchronous. But it could be the case for a null geodesic to arise from $\left(r_{b}, t_{s}\right)$ and go into the external space. This situation can be studied only after we join, at the shell $r_{b}$, the solutions found in Section 2 with an exterior solution. This will be done in Section 4 .

Remark 3.4. Note that one can conceive cases of such no trapped surface formation when pressures (2.14) do not diverge and do not remain negative in the approach to the singularity, even when w.e.c. is satisfied. Take, for instance, $\psi(a)=\sqrt[5]{a}(\sin \log a+$ $\left.\frac{5}{3}\right)$. Anyway, if pressures diverge, potential also (positively) does.

We now consider the case where $\psi$ is unbounded, positively diverging at the singularity.

Proposition 3.5. If $\lim _{a \rightarrow 0^{+}} \psi(a)=+\infty$, for any $r>0$ such that the initial data are taken outside the trapped region $\mathcal{T}$, the shell labelled $r$ becomes trapped strictly before it becomes singular, and so a black hole forms.

Proof. Fix any $r>0$. If $(r, 0) \notin \mathcal{T}$, then $\psi\left(a_{0}\right)<\frac{1}{r}$. But $\psi(a) \rightarrow+\infty$ as $a \rightarrow 0$, and therefore by continuity there must exists a time $t_{h}(r)<t_{s}$ such that $\psi\left(a\left(t_{h}(r)\right)\right)=$ $1 / r$. Then, the apparent horizon lies below the singularity curve $t=t_{s}$, which is therefore covered for any $r>0$.

Similar arguments to those made in Remark 3.3 show that, even if $\lim _{r \rightarrow 0^{+}} t_{h}(r)=$ $t_{s}$, there cannot exist future pointing null geodesic below the horizon arising from the centre, and then $t=t_{s}$ is covered at each shell.

Remark 3.6. Note that hypothesis of Proposition 3.5 does not cover all cases of unbounded $\psi(a)$, which indeed may not have limit as $a \rightarrow 0^{+}$.

Example 3.7. Consider the case of $\psi$ ruled by the power-law relation [8]

$$
\psi(a)=\sqrt{\frac{8 \pi}{3}} a^{\beta}, \quad \beta \in \mathbb{R},
$$

where $\beta<1$ by compatibility with (2.9). Hypotheses of Theorem 3.1 are always verified with this choice of $\psi$, therefore the model collapses in a finite amount of comoving time. Using the above equations one finds

$$
\begin{aligned}
& a(t)=\left(a_{0}^{1-\beta}-\sqrt{\frac{8 \pi}{3}}(1-\beta) t\right)^{\frac{1}{1-\beta}}, \\
& \phi(a)=\phi_{0}+\sqrt{\frac{1-\beta}{4 \pi}} \log \frac{a_{0}}{a}, \\
& V(\phi)=V_{0} e^{\sqrt{16 \pi(1-\beta)}\left(\phi-\phi_{0}\right)} .
\end{aligned}
$$


If $\beta$ is not negative we are in the case of Proposition 3.2 and no apparent horizon forms if one takes $r_{b}$ sufficiently small. On the other side, $\beta$ negative implies apparent horizon existence (Proposition 3.5), and

$$
t_{h}(r)=t_{s}-\gamma r^{\frac{\beta-1}{\beta}},
$$

where $\gamma>0$ depends on $\beta$. Note that the centre gets trapped at singular comoving time $t_{s}$. The particular case $\beta=-\frac{1}{2}$ corresponds - as easily inferred using (2.4) to vanishing stresses, and in fact the solution is formally equivalent to the case of homogeneous dust cloud collapse (Oppenheimer-Snyder model [16]).

\section{THE EXTERIOR REGION}

Our aim is to join the scalar field spacetime with an exterior solution at $r=r_{b}$, and study singularity arising from $r=r_{b}, t=t_{s}$. The matching will be performed along a hypersurface $\Sigma$ using Israel-Darmois junction conditions [4, 11], that requires continuity across $\Sigma$ of the first and the second fundamental forms induced on $\Sigma$ by the two spacetimes. For this reason, one cannot expect to perform the junction with Schwarzschild line element, since radial stresses of the scalar field ball do not vanish in principle (see (2.14)). Therefore, as exterior solution, we will consider the anisotropic generalization of de Sitter spacetime - see [6] and references therein that we here briefly review.

Consider a spherically symmetric spacetime such that the metric tensor and the energy-momentum tensor respectively reads

$$
\begin{aligned}
& \mathrm{d} s^{2}=-e^{2 \nu} \mathrm{d} t^{2}+e^{2 \lambda} \mathrm{d} r^{2}+Y^{2}\left(\mathrm{~d} \theta^{2}+\sin ^{2} \theta \mathrm{d} \phi^{2}\right), \\
& T_{\rho}^{\mu}=\operatorname{diag}\left(-\epsilon(r, t), p_{r}(r, t), p_{t}(r, t), p_{t}(r, t)\right) .
\end{aligned}
$$

As well known, stresses are isotropic in case of de Sitter solution $p_{r}=p_{t}=p$, where $\epsilon+p=0$. One may try to look for generalizations of this case, braking the isotropy conditions, that is supposing $p_{r} \neq p_{t}$, but still assuming

$$
\epsilon+p_{r}=0 \text {. }
$$

This amounts to weaken the degeneracy hypothesis on the tensor $T_{\nu}^{\mu}$ of de Sitter solution, retaining two different degenerate subspaces. Misner-Sharp mass reads

$$
m=\frac{Y}{2}\left[1-\left(Y^{\prime} e^{-\lambda}\right)^{2}+\left(\dot{Y} e^{-\nu}\right)^{2}\right],
$$

(in this Section, dot and prime will denote differentiation w.r.t $t$ and $r$, respectively). Using Einstein equations together with assumption (4.3) one shows that both $\epsilon$ and $m$ can be seen as functions of $Y$. In particular $m(Y)=4 \pi \int_{0}^{Y} \epsilon(\sigma) \sigma^{2} \mathrm{~d} \sigma+m_{0}$. With the additional ansatz that

$$
\left(Y^{\prime} e^{-\lambda}\right), \quad\left(\dot{Y} e^{-\nu}\right)
$$

are functions of the variable $Y$ only, that amounts to require a higher degree of symmetry of the solution - a $G_{4}$ group of motion - it can be seen that a suitable coordinate change exists, that brings the solution in the form

$$
\mathrm{d} s^{2}=-\chi(Y) \mathrm{d} T^{2}+\chi(Y)^{-1} \mathrm{~d} Y^{2}+Y^{2} \mathrm{~d} \Omega^{2}, \quad \chi(Y)=1-\frac{2 M(Y)}{Y},
$$


thereby obtaining a family of solution as the mass $M(Y)$ varies. This function $M(Y)$ is arbitrary but, to satisfy weak energy conditions, it must obey to the following law

$$
M^{\prime}(Y) \geq 0, \quad M^{\prime \prime}(Y)-\frac{2}{Y} M^{\prime}(Y) \leq 0
$$

The family outlined here contains, for instance, Minkowski, Schwarzschild, ReissnerNordström and de Sitter spacetimes as particular cases, corresponding to choosing the function $M(Y)$ respectively equal to 0 , to $m_{0}$ (constant), to $m_{0}+e_{0}^{2} / Y\left(m_{0}, e_{0}\right.$ constant) and to $\frac{4}{3} \epsilon_{0} \pi Y^{3}$. Computation of Kretschmann scalar implies that the only singularity may arise at $Y=0$ - for instance, it suffices that $m(Y)=Y^{\alpha}+o\left(Y^{\alpha}\right)$ for $Y \rightarrow 0$, with $0<\alpha<3$.

4.1. Matching conditions. Let us now consider a spherical symmetric source

$$
\mathrm{d} s^{2}=-e^{2 \nu} \mathrm{d} t^{2}+e^{2 \lambda} \mathrm{d} r^{2}+R^{2}(r, t)\left(\mathrm{d} \theta^{2}+\sin ^{2} \theta \mathrm{d} \phi^{2}\right),
$$

Our aim is to show that, at a spherical junction hypersurface $r=r_{b}$, no conditions other than continuity of the mass function are required to perform the matching. Indeed, the following proposition holds.

Proposition 4.1. A general spherical line element (4.7) can be joined with the spacetime (4.5) at a hypersurface $\Sigma:=\left\{\left(t, r=r_{b}, \theta, \phi\right)\right\}$. The matching conditions at $\Sigma$ read

$$
Y(t)=R\left(r_{b}, t\right), \quad \frac{d T}{d t}(t)=\frac{R^{\prime}\left(r_{b}, t\right)}{\chi\left(m\left(r_{b}, t\right)\right) e^{(\lambda-\nu)\left(r_{b}, t\right)}},
$$

where $m(r, t)$ is the Misner-Sharp mass function of the metric (4.7).

Proof. Let us parameterize $\Sigma$ with coordinates $(\tau, \theta, \phi)$. The injection of $\Sigma$ into the internal space equipped with metric (4.7) simply reads $(\tau, \theta, \phi) \hookrightarrow\left(\tau, r_{b}, \theta, \phi\right)$. Consequently, the fundamental forms induced by the metric on $\Sigma$ can be computed as

$$
\begin{aligned}
& I_{\Sigma}^{\text {int }}=-e^{2 \nu} \mathrm{d} \tau^{2}+R^{2} \mathrm{~d} \Omega^{2}, \\
& I_{\Sigma}^{i n t}=-e^{\lambda}\left[e^{2(\nu-\lambda)} \nu^{\prime} \mathrm{d} \tau^{2}-e^{-2 \lambda} R^{\prime} R \mathrm{~d} \Omega^{2}\right] .
\end{aligned}
$$

where we suppose the metric terms calculated in $\left(r_{b}, t\right)$.

We perform the same operation with the external metric (4.5). The injection reads in coordinates $(\tau, \theta, \phi) \hookrightarrow(T(\tau), Y(\tau), \theta, \phi)$, where $T(\tau), Y(\tau)$ are unknown functions. First fundamental form in this case is

$$
I_{\Sigma}^{e x t}=\left(-\chi(Y) \dot{T}^{2}+\frac{\dot{Y}^{2}}{\chi(Y)}\right) \mathrm{d} \tau^{2}+Y^{2} \mathrm{~d} \Omega^{2},
$$

where, with a slight abuse of notation, we denote derivatives of $T$ and $Y$ by $\dot{T}$ and $\dot{Y}$. Continuity of the first fundamental form at $\Sigma$ implies

$$
Y(\tau)=R\left(\tau, r_{b}\right)
$$

which is the first equation in (4.8), and

$$
-\chi(Y) \dot{T}^{2}+\frac{\dot{Y}^{2}}{\chi(Y)}=-e^{2 \nu} .
$$


The second fundamental form reads $\left(M_{, Y}\right.$ is the derivative of $M(Y)$ )

$$
\begin{aligned}
I_{\Sigma}^{e x t}=e^{-\nu}\{\dot{Y}[\ddot{T}+ & \left.2\left(\frac{M}{Y^{2}}-\frac{M_{, Y}}{Y}\right) \frac{\dot{Y} \dot{T}}{\chi(Y)}\right] \mathrm{d} \tau^{2}- \\
& \left.-\dot{T}\left[\ddot{Y}+\left(\frac{M}{R^{2}}-\frac{M_{, Y}}{Y}\right) e^{2 \nu} \mathrm{d} \tau^{2}-Y \chi(Y) \mathrm{d} \Omega^{2}\right]\right\},
\end{aligned}
$$

where we have used (4.13). Comparison of the angular terms in the two second fundamental forms immediately yields

$$
\dot{T}=\frac{R^{\prime}}{\chi e^{(\lambda-\nu)}}
$$

i.e. the second condition in (4.8). Finally, let us show that there are no other conditions to be imposed. First, substituting (4.8) in (4.13) we get the identity

$$
\chi(R)=\left(R^{\prime} e^{-\lambda}\right)^{2}-\left(\dot{R} e^{-\nu}\right)^{2},
$$

that only says that the mass function must be continuous across $\Sigma$, that is $m\left(r_{b}, t\right)=$ $M(Y(t))$. Therefore, we are only left with the proof that coefficients of $\mathrm{d} \tau^{2}$ in the two second fundamental forms coincide, that is we have to prove

$$
e^{-\nu}\left[\ddot{T} \dot{Y}-\dot{T} \ddot{Y}\left(\frac{M}{Y^{2}}-\frac{M_{, Y}}{Y}\right)\left(\frac{2}{\chi} \dot{Y}^{2} \dot{T}-e^{2 \nu} \dot{T}\right)\right]+e^{2 \nu-\lambda} \nu^{\prime}=0
$$

But this equation can be easily shown to hold identically by calculating the quantities $\ddot{T}$ from (4.15) and $\ddot{Y}$ from (4.16), and using Einstein equation $\dot{R}^{\prime}-\dot{\lambda} R^{\prime}-\nu^{\prime} \dot{R}=0$.

Remark 4.2. The anisotropic generalizations of de Sitter spacetime (4.5) are formally a subclass of the so-called generalized Vaidya solutions [9, 21], given by

$$
\mathrm{d} s^{2}=-\left(1-\frac{2 M(V, Y)}{Y}\right) \mathrm{d} V^{2}-2 \mathrm{~d} Y \mathrm{~d} V+Y^{2} \mathrm{~d} \Omega^{2} .
$$

Indeed, in case $M$ depends only on $Y$, i.e. $M_{, V}=0$, the coordinate transformation $\mathrm{d} V=\mathrm{d} T-\frac{1}{\chi(Y)} \mathrm{d} Y$ brings (4.5) into (4.18).

Of course, a similar result to Proposition 4.1 can be proved for this wider class of solutions. But, in this case, there is an additional condition on the mass function, that must satisfy the requirement (see also [8, equation (39)])

$$
M_{, V}=0 \text { on } \Sigma \text {, }
$$

to perform the matching with the general spherical symmetric line element 4.77.

\section{NAKED SINGULARITIES EXISTENCE}

We now confine ourselves to the case when $\psi(a)$ is a bounded function of $a$. The singularity that develops is therefore massless, as one can easily infer from (2.12) using (2.10). Moreover, in view of Proposition 3.2, the singular curve $t=t_{s}$ does not get trapped if one take $r_{b}$ sufficiently small.

In view of the results of Section 4 above, we consider the spacetime where the interior scalar field is matched with an exterior anisotropic generalization of de Sitter spacetime (4.5) at the shell $\Sigma$ labeled $r_{b}$. Let us recall that the mass (2.12) $\mathrm{m}$ is equal, 
using (2.10), to $\frac{1}{2} r^{3} a \psi^{2}(a)=\frac{1}{2} r^{2} R \psi^{2}\left(\frac{R}{r}\right)$, and therefore a necessary condition must be

$$
M(Y)=\frac{1}{2} r_{b}^{2} Y \psi^{2}\left(\frac{Y}{r_{b}}\right), \quad \forall Y \in\left[0, a_{0}^{2} r_{b}^{2}\right] .
$$

In principle, the matching condition fixes the mass function for the external region for the portion of junction surface corresponding to the observed scalar field collapsing ball, that is for $t$ running from 0 to the time of collapse $t_{s}$. For bigger values of $Y$ $\left(Y>a_{0}^{2} r_{b}^{2}\right.$ ), the mass is determined by the form of the metric for $t<0$.

As we already know, the exterior solution may possess a singularity at $Y=0$, depending on the mass profile. From the equation of state (4.3), energy diverges at $Y=0$ if $p_{r}$ does, and since, with the above choice of mass, radial pressure is continuous across junction surface, then $Y=0$ is a singularity if

$$
\lim _{a \rightarrow 0^{+}} p(a)=-\infty
$$

where $p(a)$ is the quantity, depending on $\psi(a)$, given by (2.14).

Remark 5.1. Weak energy condition (4.6) is equivalent, with the above choice of $M(Y)$, to the following two conditions on the function $\psi$ :

$$
\psi(a)+2 a \psi^{\prime}(a) \geq 0, \quad \psi^{2}(a)-a^{2}\left(\psi^{\prime 2}(a)+\psi(a) \psi^{\prime \prime}(a)\right) \geq 0 .
$$

We observe by the way that the first of these inequalities implies that potential (2.16) diverges. Conditions (5.3) are satisfied by power-law models of Example 3.7 when $\psi$ is bounded, i.e. for non-negative $\beta$.

We prove the following theorem.

Theorem 5.2. Suppose that $\psi(a)$ is bounded and satisfies (5.2), and let $r_{b}$ such that $1-\psi^{2}(a(t)) r^{2}$ is bounded away from 0 uniformly on $\left(0, r_{b}\right) \times\left(0, t_{s}\right)$. Then, the boundary $\Sigma=\left\{r=r_{b}\right\}$ of the scalar field collapses to a naked singularity.

Proof. Equations (4.8) becomes, using (2.10), (2.12) also,

$$
Y(t)=a(t) r_{b}, \quad \frac{\mathrm{d} T}{\mathrm{~d} t}(t)=-\frac{r_{b}}{1-r_{b}^{2} \psi^{2}(a(t))} .
$$

Let $r_{b}$ such that $1-\psi^{2}(a(t)) r^{2}$ is bounded away from 0 . Such a choice is, of course, always made possible because $\psi(a)$ is bounded. Then $\frac{r_{b}}{1-r_{b}^{2} \psi^{2}(a(t))}$ is bounded in $\left(0, a_{0}\right)$ and we can integrate the ODE in (5.4) to obtain

$$
\lim _{t \rightarrow t_{s}} T(t)=T_{0} \in(0,+\infty)
$$

Then the anisotropic generalization of de Sitter spacetime solution can be extended up to $T=T_{0}, Y=0$. But in a neighborhood of this point, $\chi$ is bounded away from zero because junction conditions (5.4) imply continuity of the mass function, and by hypothesis, $1-\frac{2 m\left(t, r_{b}\right)}{Y\left(t, r_{b}\right)}$ is bounded away from zero as the singularity is approached. Then the ODE

$$
\frac{\mathrm{d} T}{\mathrm{~d} Y}=\frac{1}{\chi(Y)}
$$

can be solved in a right neighborhood of $Y=0$, and there exists a radial null geodesic starting from the singularity, which is therefore naked. 
Remark 5.3. The above theorem applies to cases described in Example 3.7 with $\beta \geq$ 0 . We stress the fact that, in the approach to the singularity $t=t_{s}, \frac{2 m}{R}=\psi(a(t))^{2} r_{b}^{2}$ does not need to vanish, but may also tend to a constant nonzero value, or may even not possess a limit value. Consider, for instance, Example 3.7 with $\beta=0$. We know that that no horizon forms if $r_{b}$ is sufficiently small, but now $1-\frac{2 m}{R}$ is a positive constant on $\Sigma$.

5.1. Strength of the singularity. Since we have found examples of spacetimes exhibiting a naked singularity, it may be of interest to determine its strength. The first definition of strong curvature singularity was suggested by Tipler [19] and studied e.g. in [3, 20]. Modifications to this definition have been also suggested by Nolan [14] and Ori [17] to take into account pathological situations where, although volume forms along geodesics preserve, Jacobi fields have opposite irregular behavior - i.e. one diverges and another one vanishes in the approach to the singularity. For most of our purposes, anyway, it will suffice to look at the behavior of the quantity $k^{2} \Psi=k^{2} R_{\alpha \beta} K^{\alpha} K^{\beta}$ along causal geodesics, where $R_{\alpha \beta}$ is Ricci tensor, $K^{\alpha}=\frac{\mathrm{d} x^{\alpha}}{\mathrm{d} k}$ is the tangent vector of the geodesic with parameter $k$. If this quantity remains bounded away from zero in the approach to the singularity, this one may be considered as physically meaningful.

Let us first consider the metric (2.2). If $K^{\alpha}$ is a radial null geodesic, it can be computed, using (2.10), that

$$
k^{2} \Psi=2\left(\frac{k}{a} \frac{\mathrm{d} a}{\mathrm{~d} k}\right)^{2}\left[1-\frac{\psi^{\prime}(a) a}{\psi(a)}\right]
$$

If the limit $\frac{\mathrm{d} a}{\mathrm{~d} k}$ exists as $k \rightarrow 0$, the quantity in round brackets tends to a finite value, while the quantity in square brackets remains bounded away from zero if $\epsilon+p>0$ (see (5.1)), and then the radial null geodesic terminates in a strong singularity.

Of course, in our model the interior scalar field region is matched with an exterior where metric tensor is given by (4.5), and so strength of the central singularity of (4.5) must be determined to see whether strong singularity persists.

Proposition 5.4. If, $\forall a \in\left(0, a_{0}\right)$, the quantity $\left(\frac{a \psi^{\prime}(a)}{\psi(a)}+\frac{a \psi^{\prime \prime}(a)}{\psi^{\prime}(a)}+2\right)$ is well defined and bounded away from zero, then there exists causal geodesics terminating into the naked singularity and satisfying the limit strong curvature condition $\lim _{\inf } \operatorname{lin}_{k \rightarrow 0}\left|k^{2} \Psi\right|>$ 0 .

Proof. Let us evaluate the strength of the $Y=0$ singularity in this case, recalling that the mass $M(Y)$ is given by (5.1). Actually, it can now be checked that $\Psi$ vanishes along null geodesics, and so we will consider the situation for timelike radial geodesics. Their equations integrate to give (see also (5.4))

$$
\frac{\mathrm{d} T}{\mathrm{~d} k}=\frac{\xi}{\chi(Y(k))}, \quad \frac{\mathrm{d} Y}{\mathrm{~d} k}=\sqrt{\xi^{2}-\zeta^{2} \chi(Y(k))},
$$


( $\xi$ and $\zeta \neq 0$ are integration constants) that is, they are regular at $Y=0$. Therefore, considering a geodesic with $\frac{\mathrm{d} Y}{\mathrm{~d} k} \rightarrow 0$, it can be seen that

$$
\begin{aligned}
\left|k^{2} \Psi\right|=\frac{k^{2}}{Y} M^{\prime \prime}(Y) & =\frac{2}{\mathrm{~d}^{2} Y / \mathrm{d} k^{2}} M^{\prime \prime}(Y)= \\
& 2 \zeta^{-2} \frac{M^{\prime \prime}(Y) Y^{2}}{Y M^{\prime}(Y)-M(Y)}=2 \zeta^{-2}\left(2+\frac{a \psi^{\prime}(a)}{\psi(a)}+\frac{a \psi^{\prime \prime}(a)}{\psi^{\prime}(a)}\right),
\end{aligned}
$$

and the proposition is proved since last term is bounded away from zero.

Example 5.5. The above theorem applies to the power-law cases discussed in Example 3.7 with $\beta$ strictly positive, and more generally for functions $\psi$ not-decreasing but strictly concave in $\left(0, a_{0}\right)$.

The case $\beta=0$ (see also Remark $\left[5.3\right.$ ), where $\left|k^{2} \Psi\right|$ is seen to go to zero, may be treated using ideas from [14], and results in a weak singularity.

\section{DISCUSSION AND CONCLUSIONS}

As we have seen, the formation of singularities the gravitational collapse of homogeneous scalar fields with potential is completely ruled by a condition of integrability of a function related to the energy density of the model. If this function is bounded, apparent horizon formation is avoided during the evolution, and therefore the singularity is massless, which, as is well known, is actually a feature of any spherically symmetric naked singularity.

The boundary develops a naked singularity when the exterior solution is given by anisotropic de Sitter generalization (4.5), and the singularity turns out to satisfy a strong curvature condition for a class of examples that also includes power-law cases studied in [8].

Let us briefly comment on the choice of the exterior region. In the examples discussed in [8], the matching is performed with a wider class, that is the generalization of Vaidya spacetime. One may wonder whether the strong curvature condition is satisfied also along null geodesics with this more general choice of mass profile. Actually, in this case $k^{2} \Psi$ is not identically zero - as it happens in the case we studied - but it can be checked that again it vanishes in the limit $k \rightarrow 0^{+}$, due to matching condition 4.19.

It must therefore be observed that, if the exterior region is given by (4.5) or even the more general metric (4.18), the limit strong curvature condition proved in Proposition 5.4 does not hold for any radial geodesic, but only for timelike geodesic satisfying $\frac{\mathrm{d} Y}{\mathrm{~d} k} \rightarrow 0$ in the approach to the singularity. Such a case is possible due to the fact that no apparent horizon form in the interior region, and $\chi$ is bounded away from zero near the singularity. Although one may try and see what happens with other exterior metrics, this fact seems to be a distinctive feature of the homogeneous scalar field model under exam, which possess a synchronous singularity, and therefore is qualitatively different from other Cosmic Censorship counterexamples known in literature [5, 7].

In conclusion, breaking the homogeneity assumption of scalar field models with potential seems an unavoidable step to retain strong curvature condition along any causal geodesic terminating into the naked singularity. 


\section{REFERENCES}

[1] M. Choptuik, Phys. Rev. Lett. 70 (1993) 9

[2] D. Christodoulou, Ann. Math. 140 (1994) 607

[3] C. J. S. Clarke and A. Krolak, J. Geom. Phys. 2 (1985) 127

[4] G. Darmois, Mémorial des Sciences Mathmatiques Gauthier-Villars, Paris, 1927, Vol. 25.

[5] I. H. Dwivedi and P. S. Joshi, Comm. Math. Phys. 166117 (1994).

[6] R. Giambò, Class. Quantum Grav 19 (2002) 4399

[7] R. Giambò, F. Giannoni, G. Magli, P. Piccione, Class. Quantum Grav., 20, L75 (2003)

[8] R. Goswami, P. S. Joshi, gr-qc/0410144

[9] K. S. Govinder and M. Govender, Phys.Rev. D 68 (2003) 024034,

[10] T. Hertog, G. Horovitz, and K. Maeda, Phys. Rev. Lett. 92 (2004) 131101;

[11] W. Israel, Nuovo Cim. 44B (1966) 1, Nuovo Cim. 49B (1966) 463 (erratum)

[12] D. Kramer, H. Stephani, E. Herlt, M. MacCallum, Exact Solutions of the Einstein's Field Equations, Cambridge University Press (1980)

[13] G. Magli, Class. Quantum Grav. 14 (1997) 1937-1953

[14] B. C. Nolan, Phys. Rev. D 60 (1999) 024014

[15] N. J. Nunes, J. E. Lidsey, Phys.Rev. D 69 (2004) 123511

[16] J. R. Oppenheimer, and H. Snyder, Phys. Rev. 56 (1939) 455

[17] A. Ori, Phys. Rev. D 61 (2000) 064016

[18] R. Penrose, Nuovo Cimento 1252 (1969).

[19] F. J. Tipler, Phys. Lett. 64A (1987) 8

[20] F. J. Tipler, C. J. S. Clarke, and G. F. R. Ellis, in General Relativity and Gravitation Vol. 2 (ed. A Held), Plenum NY (1980)

[21] A. Wang, Y. Wu, Gen. Rel. Grav. 31 (1999), 107.

DiPARTIMENTO Di MATEMATICA E INFORMATICA, UNIVERSiTÀ Di CAMERINO, ITALY

E-mail address: roberto.giambo@unicam.it

URL: http: //dmi.unicam.it/ giambo 